(C) by R. Oldenbourg Verlag, München

\title{
Crystal structure of barium tristannide, $\mathrm{BaSn}_{3}$
}

\author{
R. Kröner, K. Peters and H. G. von Schnering
}

Max-Planck-Institut für Festkörperforschung, Heisenbergstraße 1, D-70569 Stuttgart. Germany

Received March 4, 1998. transferred to Ist update of database ICSD in 1999, CSD-No. 409263
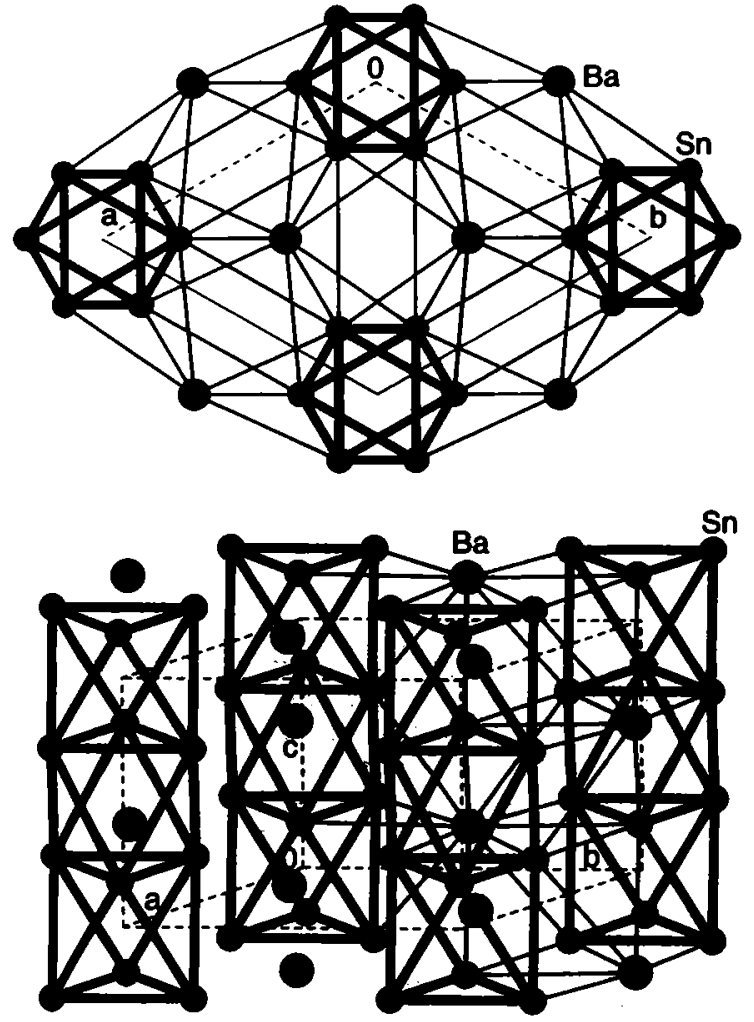

Source of material: Single crystals of BaSnz were formed in the synthesis of ternary E13/E14 clathrates with Ba. Mixtures of the elements were loaded under $\mathrm{Ar}$ into $\mathrm{Nb}$ or $\mathrm{Ta}$ capsules. The sealed capsule was heated up to $1270 \mathrm{~K}(3 \mathrm{~h})$, annealed at $970 \mathrm{~K}(2-3 \mathrm{~d})$ and cooled down (see ref. 1).

The existence of the BaSn3 phase is known for a long time but the structure was not determined (see ref. 2). BaSn3 forms the $h P 8$ structure $\mathrm{Ni}_{3} \mathrm{Sn}$ with $\mathrm{Ba}$ and $\mathrm{Sn}$ at the positions of $\mathrm{Sn}$ and $\mathrm{Ni}$, respectively. Important interatomic distances: $\mathrm{Ba}-\mathrm{Sn}=3.663 \AA$ $(6 \mathrm{x}), 3.641 \AA(6 \mathrm{x}) ; \mathrm{Sn}-\mathrm{Sn}=3.058 \AA(2 \mathrm{x}), 3.266 \AA(4 \mathrm{x})$, and $\mathrm{Sn}-\mathrm{Ba}=3.641 \AA(2 \mathrm{x}), 3.663 \AA(2 \mathrm{x})$. Very recently the $\mathrm{BaSn}_{3}$ structure was also determined by Fässler and Kronseder (see ref. 3), who knew our results. Both determinations are in agreement.

BaSn3, hexagonal, $P 63 / m m c$ (No. 194), $a=7.253(2) \AA, c=5.496(2) \AA$, $V=250.4 \AA^{3}, Z=2, R(F)=0.021, R_{W}(F)=0.019$.

Table 1. Parameters used for the X-ray data collection

$\begin{array}{ll}\text { Crystal: } & \text { silvery parallelepiped, size } 0.3 \times 0.2 \times 0.1 \mathrm{~mm} \\ \text { Wavelength: } & \text { Mo } K_{\alpha} \text { radiation }(0.71069 \AA) \\ \mu: & 209.4 \mathrm{~cm}^{-1} \\ \text { Diffractometer: } & \text { SYNTEX PI } \\ \text { Scan mode: } & \omega \\ T_{\text {measurement: }} & 293 \mathrm{~K} \\ 2 \theta_{\text {max: }} & 55^{\circ} \\ \text { N }(h k)_{\text {unique: }} & 132 \\ \text { Criterion for } I_{\mathrm{o}}: & I_{\mathrm{o}}>3 \sigma\left(I_{0}\right) \\ \text { N(param) } & 8 \\ \text { Programs: } & \text { SHELXTL-plus, SHELXS-86, SHELX-76 }\end{array}$

Table 2. Final atomic coordinates and displacement parameters (in $\AA^{2}$ )

\begin{tabular}{lllllllllll}
\hline Atom & Site & $x$ & $y$ & $z$ & $U_{11}$ & $U_{22}$ & $U_{33}$ & $U_{12}$ & $U_{13}$ & $U_{23}$ \\
\hline Ba & $2 c$ & $1 / 3$ & $2 / 3$ & $1 / 4$ & $0.0220(7)$ & $0.0220(7)$ & $0.0213(5)$ & 0.022 & 0 & $0.0000(7)$ \\
Sn & $6 h$ & $0.8594(1)$ & $1.7188(1)$ & $1 / 4$ & $0.0263(5)$ & $0.0180(4)$ & $0.0175(4)$ & $0.0180(4)$ & 0 & 0 \\
\hline
\end{tabular}

\section{References}

1. Kröner, R.: Zintl-Phasen der Alkalimetalle und des Bariums mit Clathratstruktur. Dissertation, Universität Stuttgart, 1989.

2. Ray, K. W.; Thompson, R. G.: Metals Alloys 1 (1930) 314.

3. Fässler, T. F.; Kronseder, C.: BaSn3, ein Supraleiter im Grenzbereich zwischen Zintl-Phasen und intermetallischen Verbindungen: Realraumanalyse von Bandstrukturen. Angew. Chem. 109 (1997) 2800-2803.
4. Sheldrick, G. M.: Program Package SHELXTL-plus. Release 4.1. Siemens Analytical X-Ray Instruments Inc., Madison(WI 53719), USA 1990.

5. Sheldrick, G. M.: Phase Annealing in SHELLX-90: Direct Methods for Large Structures. Acta Crystallogr. A46 (1990) 467-473.

6. Sheldrick, G. M.: SHELLI-76, Programs for Crystal Structures Determination. Cambridge 1976 